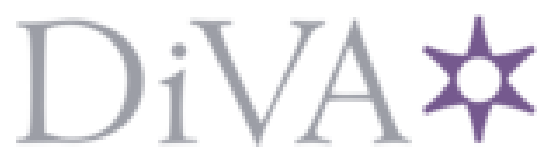

http://www.diva-portal.org

\title{
Postprint
}

This is the accepted version of a paper published in Tizard Learning Disability Review. This paper has been peer-reviewed but does not include the final publisher proof-corrections or journal pagination.

Citation for the original published paper (version of record):

Adolfsson, P., Ek, P., Umb-Carlsson, Õ. (2019)

Dietitians' challenges when consulting to adults with intellectual disabilities

Tizard Learning Disability Review, 24(4): 153-162

https://doi.org/10.1108/TLDR-11-2018-0033

Access to the published version may require subscription.

N.B. When citing this work, cite the original published paper.

Permanent link to this version:

http://urn.kb.se/resolve?urn=urn:nbn:se:uu:diva-395125 


\section{Dietitians' challenges when consulting to adults with intellectual disabilities}

Today, in many countries, adults with intellectual disabilities (ID) live in the community in their own housing. Such individuals often require lifelong support with regard to housework, finances and other everyday activities (Umb-Carlsson and Sonnander, 2005). Nutritional problems such as obesity are relatively common among adults with ID (e.g. Adolfsson et al., 2008; Casey and Rasmussen, 2015) as well as an increased prevalence of underweight and deficient nutrition (Adolfsson et al., 2008; Ptomey and Wittenbrook, 2015). Such conditions often result in secondary health problems, such as diabetes, osteoporosis and cardiovascular disease (Cheetham and McMillan, 2017; NG et al., 2017). The health issues of adults with ID are seldom included in the education of healthcare professionals, and therefore, their knowledge of the health issues of adults with ID is often insufficient (Humphries et al., 2012; Ptomey and Wittenbrook, 2015; Davies and Lennox, 2014).

To counteract the nutritional problems of adults with ID, registered dietitians (RDs) with relevant experience should be engaged in their health care (Ptomey and Wittenbrook, 2015; Davies and Lennox, 2014). Humphries et al. (2012) studied RDs working in group homes for adults with ID in the USA. Several States require that group homes use RDs to supervise food-related tasks. However, these services are not always addressed at an individual level; instead, an RD plans one menu that is used by numerous group homes for all residents, without considering the different circumstances of individuals. The researchers note that, to serve adults with ID, RDs need to have additional training beyond the standard dietetic education, which 53 per cent of the respondents had received (Humphries, et al. 2012).

Few studies have been carried out on the general working conditions of RDs. Devine et al. (2004) studied RDs who mainly work with individual nutrition services at the community level. They found that RDs experienced frustration when other health workers and employers did not understand that a dietary change process is intensive, needs to be individualised and takes a long time to fully implement. RDs reported a lack of respect and difficulty in meeting the expectations of both their clients and co-workers. However, RDs experienced satisfaction and identified themselves as experts when their work had positive impacts on their clients and co-workers (Devine et al., 2004). 
There is insufficient knowledge about RDs' work with adults with ID, which has not previously been studied in Sweden. Therefore, the aim of the present study was to describe RDs' experiences in advising adults with ID in Sweden.

\section{Method}

A descriptive study using a web-based questionnaire was conducted. Respondents' answers to open-ended questions were analysed.

The Regional Ethical Review Board of Uppsala (Dnr 2017/224) approved the study design.

\section{Respondents}

This study included RDs with experience of advising adults with ID regarding nutritionrelated health promotion. First, all 21 Swedish regional habilitation centres were contacted to determine if any RDs were employed to advise adults with ID. In ten centres, there was at least one RD employed for that purpose $(\mathrm{N}=19)$. Second, all primary care districts in all Swedish regions were contacted by e-mail or telephone, and their web sites investigated, 130 RDs being identified. Third, using the web site of the Swedish Association of Clinical Dietitians (DHR), 31 more RDs were found. Fourth, 21 RDs were identified in a network for professionals interested in meals for adults with ID, organized by the Swedish national food agency. In total, 201 dietitians were identified as eligible for inclusion in this study.

\section{Questionnaire}

A web-based questionnaire with 22 questions was developed about RDs' working routines and experiences. The questionnaire included several multiple-choice questions and, as this was a new area of study, spaces for personal comments and open-ended questions were included (Bryman, 2008). The design enabled respondents to use their own words without researcher influence (Reja et al., 2003). The questions were developed by the authors, after discussions with disability and clinical nutrition researchers within the Department of Public Health and Caring Science at Uppsala University. Thereafter, the questionnaire was pilot tested, but no further change required. This process was done to ensure face validity (Bryman, 2008). The first questions were about gender, the area the RDs worked in, their general professional experience and the length of their professional experience with adults with ID. Further questions targeted RDs' clinical work with adults with ID: why an RD is 
consulted, who takes the initiative, what are the consulting procedures, and what are the barriers and facilitators RDs experience when trying to achieve satisfactory results.

\section{Procedure}

The 201 RDs identified were contacted by email. The email included a link to the questionnaire and information about the study. Anonymity was guaranteed as the questionnaire was administered by a survey tool. Recipients were encouraged to contact the researchers if they knew of other eligible RDs. However, none were found. Two reminders were sent before deactivating the survey link after five weeks.

\section{Data analysis}

Respondents' answers to the open-ended questions and additional comments were analysed with systematic text condensation (STC) (Malterud, 2012), a method of thematic cross-case analysis involving four steps and inspired by phenomenological ideas.

In the first step, all open-ended answers and personal comments were combined into a document, read and by all authors to obtain an overview. The authors identified four preliminary themes about the RDs' work and working conditions. In the second step, the first author organized the meaning units, coded the units and sorted the codes into code groups according to the preliminary themes. The first and third author discussed the codes and code groups, re-coding until consensus was reached. In the third step, each code group was considered an analytic unit, and the codes sorted into subgroups. Thereafter, the content of the meaning units was condensed. In the fourth step, the most salient content and meaning of each theme were determined. All authors participated in this stage. Additionally, quotations illustrating the content were chosen. Thereafter, the subgroups and code groups used to build new subcategories and categories were labelled with headings illustrating their content.

\section{Results}

In total, 53 RDs, (26 per cent of 201) comprising 52 female and one male, completed the questionnaire. A total of 29 respondents had more than five years of professional experience as a dietitian for adults with ID; 24 respondents had less than five years such experience. 
Between 22 and 47 of the RDs answered the open-ended questions. Interestingly, most (47 of 53) RDs answered the question: What are the barriers and facilitators the RDs experience when trying to achieve satisfying results through consulting?

The categories and subcategories that emerged from the respondents' comments are shown in Figure 1. Each category and subcategory will be explained and illustrated with quotations. The quotations below have numbers in brackets showing that they are made by different respondents. Comments were translated from Swedish to English to match the original essence of the quotation as closely as possible. This sometimes meant sacrificing consistent terminology (e.g., use of both "patient" and "user" to describe an adult with ID) and the quotations do not always follow grammatical rules.
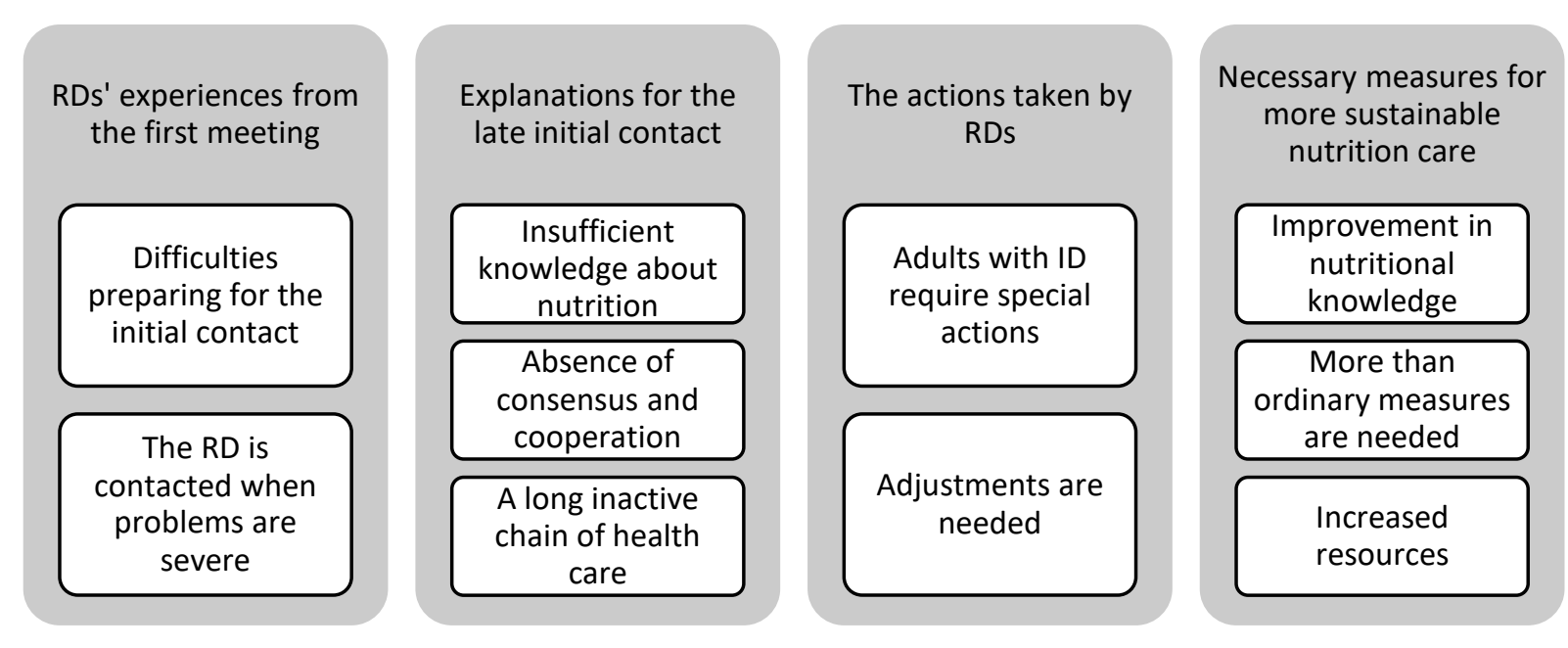

Figure 1. Identified categories and subcategories.

\section{RDs' experiences from the first meeting}

Respondents experienced difficulties preparing for the first meeting with an adult with ID. They also found that health problems were often already severe at initial contact.

\section{Difficulties preparing for the initial contact}

Respondents reported receiving inadequate information about an adult with ID before initial contact. When the appointment is made, information about the person's disability is not always provided, nor does a medical referral from a general practitioner, a nurse or other 
health professional always include necessary information about the special needs that can reflect both the cognitive and physical disabilities of the person:

It is hardly ever obvious that the referral concerns a person with ID. (5)

In general, an RD meets the adult with ID together with staff from the residence or a relative at the clinic. Having supporting staff present made it easier to communicate and compensate for the insufficient information received beforehand. Respondents had also experienced situations in which they are expected to provide a consultation without ever meeting the adult with ID, sometimes when staff judge that the adult with ID has difficulties coping with new situations:

The client is not always present, judged from the fact that the client has so many contacts, so information from me should induce stress. (21)

The respondents noted that it is also possible for an individual to contact an RD alone without any supporting staff or relative.

\section{The $R D$ is contacted when problems are severe}

According to the respondents, the most typical reasons that adults with ID need to consult with an RD are concerns about overweight/obesity, underweight and diabetes. RDs are commonly contacted when health problems are already severe:

Often experience that it has gone too far. We had been able to do more if we had intervened earlier. (23)

Only three respondents reported always being contacted in a timely manner concerning advice for adults with ID.

\section{Explanations for late initial contact}

The main reasons given by respondents for not being contacted earlier and not being informed about the special needs of adults with ID included insufficient knowledge about nutrition, a lack of consensus and cooperation among staff and a long chain of care. 


\section{Insufficient knowledge about nutrition}

Respondents suggested that a lack of knowledge about nutrition is common among adults with ID and supporting staff. Thus, staff may believe that adults with ID are able to manage food-related tasks without support. In contrast, respondents often see these adults as lacking both knowledge and ability to meet such expectations. Respondents also noted a discrepancy between the ability to manage food-related tasks and making healthy choices:

For those who live by themselves I can often experience that there is a demand that they should be able to do more than what I consider they have capacity for.

However, many adults with ID need support managing their everyday life activities and often receive support by the staff at their home. Such support regarding food and health was believed by respondents to not always be individually tailored and adequate for each person. They indicated that staff commonly do not have general knowledge about nutrition and, therefore, do not understand its importance in disease prevention:

Poor basic knowledge of diet and nutrition. Often a great interest and good will but lack of knowledge. (21)

\section{Absence of consensus and cooperation}

Respondents suggested that it may be difficult for staff with inadequate knowledge to establish common routines for healthy and nutritious choices by the persons they support:

There are no routines for preventive work, concerning both underweight and overweight/obesity. (48)

They further argue that different opinions between staff about a healthy diet may contribute to difficulty achieving a consensus. If staff cannot separate their own opinions and preferences from healthy nutritional guidance, an individual's health problems may not be identified in a timely manner, despite staff intending to provide optimal treatment to the persons they support:

Lack of flow of information in the staff at the residence. Personal preferences of the staff at the residence. (15) 


\section{A long inactive chain of health care}

A long, inactive health-care chain can prevent nutritional problems from being treated in time. The adult with ID may be incapable of contacting an RD and, therefore, depend on support. Supporting staff may not act rapidly because of a lack of knowledge. Subsequently, health care professionals may first attempt to solve the problem themselves; if they fail, they then seek to cooperate with an RD or provide a medical referral so that an RD is contacted:

It probably takes time before it is really noticed, is assessed by a physician before it eventually is referred to me. (37)

The respondents also noted that, although the health care chain can sometimes work rapidly, the chain can be broken because of a lack of RDs engaged with this group of adults:

There is too few RDs that can make the assessment in time, most often other persons point out the need and by then too long time has elapsed. (42)

\section{The actions taken by RDs}

Different actions are often required when consulting with adults with ID compared with adults in the general population. Thus, to achieve effective results, RDs need to adjust their consultation methods and courses of action.

\section{Adults with ID require special courses of action}

Respondents noted differences between consulting with adults with ID who usually receive support in their everyday life and adults in the general population. The RD needs individualised knowledge about the person with ID for a successful consultation. The RD must also ensure that nutritional advice reaches both the person to whom the advice is directed and their supporting staff. In addition, a prerequisite for implementing the advice is that the manager of the housing residence and the nurse serving the person are also involved. Respondents indicated that it is problematic if a person visits the RD alone and 
wishes that nobody else be informed of the consultation. In such cases, the RD is bound to confidentiality:

If the patient comes alone no contact is taken with other persons if it is against the patient's will. (35)

Respondents suggested that home visits by an RD are often a better alternative, especially when it is difficult for the person to visit the clinic. During home visits, the RD can obtain information about the individual's living conditions that facilitates the consultation's effectiveness. The RD also has the opportunity to observe mealtime activities, food preparation, eating routines and interaction during meals. Home visits may allow the RD to meet more than one member of staff, as opposed to when the consultation occurs at the clinic. However, home visits are time consuming and, therefore, not always feasible. Such visits are only possible if clinic operation is not disturbed. Hence, 22 (41 per cent) of the respondents did not consider it possible to make home visits.

A total of 34 (64 per cent) of the respondents reported that they follow-up consultations. They consider nutritional consultation a long-term process and that follow-ups are needed because it takes time to change food habits:

It mostly concerns change in lifestyle that takes time and that has to be followed up in order to make it a habit. (29)

At follow-ups, the RD can gain information about how their advice is working and if any adjustments are needed. Follow-ups may be carried out in different ways, and there are no commonly adopted strategies. For example, the RD may initiate a new appointment for a consultation at the clinic, make a home visit, or make a telephone call. Sometimes, the responsibility for the follow-up is left to the person or the support staff: 
It is important that a follow-up is planned in order to see how the information has been dealt with, if the nutritional problem has improved and if further contact is needed. (33)

\section{Adjustments are needed}

The respondents noted that in contact with the person with ID, the RD needs to be responsive and flexible. Different methods help to deliver information. The information can be written or oral, and can be provided to the person, to others accompanying the person, or to the whole staff group at home visits:

A long-term plan for the patient is often needed in order to make changes, but also for the staff to implement routines. (45)

One problem, according to the respondents, is the person's legal right to autonomy and selfdetermination. Their ID may obstruct the person from understanding the importance of a healthy diet so that it is difficult and time-consuming to persuade the person to make the necessary dietary changes to improve his or her health:

It is not always easy for the staff to guide the patient in the right direction, can be experienced as being pushy to warn all the time and control the shopping the patient sometimes does on his own (37)

\section{Necessary measures for more sustainable nutrition care}

The respondents emphasize requirements that are needed for sustainable nutrition of adults with ID. Nutritional knowledge among individuals with ID and their supporting staff should be improved. Respondents remarked that the measures they had to take meant that RD resources need to be increased. 
Respondents suggested that having sufficient nutritional knowledge could make it easier to follow the RD's advice and identify nutritional deficiencies. They emphasized the importance of preventive measures for adults with ID. Nutritional knowledge is also needed to increase the professionalism of the supporting staff involved in food-related decisions on a daily basis. In addition, persons who live independently need more nutritional knowledge: The person with ID can have persons around that have their own ideas about how the food should be for the person in question. It can conflict with the RD's dietary advice. (18)

Other health professionals also need increased nutritional knowledge so that they understand and are aware of the nutritional risks for adults with ID.

\section{More than ordinary measures are needed}

Irrespective of whether respondents were able to make home visits, they highlight the opportunities that these can provide. RDs can provide the same information to all the people involved, they can maintain a dialogue with staff, and, if needed, they can practice some cooking with individuals with ID or staff. When individuals are not able to visit the clinic, the possibility of home visits should be natural and perceived as an essential practice of RD work:

Have no possibility to make home visits. That would sometimes have facilitated a better understanding about the patient's situation and allowed to meet several of the staff. (36)

Respondents expressed the need for cooperation among the different professionals involved in the lives of adults with ID: staff, health care professionals and managers in support 
services. They believe that, with cooperation, compliance with advice provided through the consultation could be improved.

Regarding cooperation, the respondents included reciprocal information exchange and an understanding of the importance that all those concerned obtain the necessary information. They also stated the need for cooperation in situations where misunderstanding or confusion about the given recommendations arise so that the RD has the opportunity to address these issues:

Since you don't have the contact and can inform all the staff it will be uncertain if all information has got through as you wanted, there is no time to ask questions to confirm that the staff has understood the given advices. (37)

Cooperation with support staff is particularly important after a person with ID meets an RD alone. When the RD lacks knowledge about the person's disabilities, the consultation becomes complicated and is unlikely to result in improving the person's health.

\section{Increased resources}

Respondents requested sufficient resources to improve the health of adults with ID. For example, they reported that special scales are seldom available at clinics. Further respondents argued that RDs need more time for consultations with adults with ID than for adults in the general population. Time is needed to persuade both the person and the supporting staff to implement the necessary changes in a person's food habits. They noted that resources are needed to supply sufficient, individualised information to adults with ID. The information needs to be concrete for easy implementation and to avoid misunderstandings. To achieve successful results, the RD needs to ensure that all those involved understand and follow the advice: 
Feel that it can be difficult to give advice to persons with ID that maybe have more problems to understand the information. Want a solid and simple material or other instruments for use for these patients. (36)

Only five respondents (9 per cent) reported that information about adults with ID was included in their education. Respondents generally had limited knowledge about adults with ID, and therefore, their consultation methods were often improvised. They explained that knowledge can be acquired in different ways, e.g., written information from Swedish authorities, resources from the Internet and lectures, information received from a colleague with more experience, or learning by doing.

The respondents revealed that, in general, there is a need to increase the competence of RDs working with adults with ID. RDs require knowledge about the consequences of the ID and about the everyday life of adults with ID who are often dependent on support. Furthermore, they expressed a need for special training in communication methods and treatment:

Increased education in methods of dialog and how a setup of treatment could be designed for these patients. (11)

Maybe I could have got some kind of increased competence within the subject that had prepared me better for the job. (37)

\section{Discussion}

The results of this first study of Swedish RDs' experiences with adults with ID reveal a poorly functioning system. RDs do not receive sufficient information about the special needs of adults with ID beforehand and, therefore, are not prepared for the situation. In addition, adults with ID have severe nutritional problems that are more difficult to address, indicating that the nutrition-related health of adults with ID is typically not taken seriously. This is a remarkable finding, as it is known that nutritional problems 
are common among this group (Adolfsson et al., 2008; Humphries, et al., 2009; Casey and Rasmussen, 2015). Respondents in the present study were aware of the insufficient nutritional knowledge of support staff and other professionals, and claimed that this delays the discovery of problems and contributes to the late contact with individuals with ID.

People are generally resistant to changing their food habits (Fieldhouse, 2002). The present respondents noted that the special needs of adults with ID and the involvement of several others can hinder compliance with the RD's advice. It seems to be difficult for RDs to deliver the information to all those concerned. Health promotion in general for adults with ID needs to consider the effects of the environment, according to Marks and Sisirak (2014); a supportive environment needs to be developed for sustainable and comprehensive health promotion. According to them, several theories can be applied, such as the theory of diffusion of innovation. Additionally, Fieldhouse (2002) noted that the idea of changing food habits should be seen as an innovation in order to achieve success. The diffusion of innovation theory describes changing attitudes and practices in a social system (Rogers, 2003); it contains five attributes that need to be considered to implement a new practice: relative advantage, compatibility, complexity, trialability and observability. The categories in the present study (Figure 1) are discussed in relation to these five attributes.

For relative advantage a new practice needs to be more positive and better than the practice to be changed (Rogers, 2003). In the present study, adults with ID and their networks did not always understand the presence of nutritional problems. Thus, it was challenging for an RD to explain the harm and problems associated with certain practices and existing food habits and to determine methods to encourage change. Respondents reported that time and resources in addition to those already available to RDs are needed. In the present study this was exemplified by the category "Necessary measures for more sustainable nutrition care"; these requirements are generally in line with the recommendations suggested by Humphries et al. (2009).

According to Rogers (2003), a new practice that is not consistent with a person's norms and values can be difficult to adopt. A new practice needs to be compatible with existing values 
and must be feasible to implement by an adult with ID, whose everyday life may differ from those of the general population. In the present study, contact with an RD was often made late when problems were already severe. The category "Explanations for the late initial contact" indicates that existing norms and values are often based on a lack of knowledge about nutrition and a lack of attention to nutritional problems, that can negatively influence implementation of new practices. These reports by the respondents are similar to the frustrating experiences noted by Devine et al. (2004) in their study of RDs working in the community. It is critical that RDs cooperate with adults with ID and their supporting staff. They should consult with the individual's support staff, as their knowledge of the person they support usually is comprehensive (Cheetham and McMillan, 2017). Information needs to be transferred to in both directions between dietitians and staff to facilitate the adoption of the new practice. Humphries et al. (2009) recommended that RDs should be part of interdisciplinary teams surrounding adults with ID. However, to increase attention to nutritional problems, an assessment instrument should be developed for this group; there are few assessment methods for nutritional behaviour in adults with ID (Casey and Rasmussen, 2015).

Complexity is the attribute that addresses the degree of difficulty of a new practice. If the innovation is difficult to understand and implement, it is less likely that it will be adopted or adoption will take longer (Rogers, 2003). The present respondents reported that the health problems of adults with ID were often severe by the time RDs were contacted. The category "RDs' experiences of the first meeting" indicated that the required measures need to be complex. However, changing food habits is often difficult, and is easier to accomplish if the required effort is small (Fieldhouse, 2002). To counteract the complexity, RDs need to be proactive, facilitate implementation, and provide persons with ID and supporting staff with easily understandable information (Humphries et al., 2009). However, adults with ID live in special social contexts with different ways of organizing their daily meals (Adolfsson et al. 2012). RDs need to take into account how to reduce the complexity of meal organization to facilitate the implementation of new practices.

The fourth attribute of the theory is trialability. To make it is easier to adopt new practices, extensive innovations that greatly influence a person's life should be introduced in trialable and limited parts (Rogers, 2003). Fieldhouse (2002) argued that innovations in smaller parts 
contain less risk and, therefore, are easier to adopt. "The actions taken by RDs" category revealed that RDs need to implement special measures and remain in contact with adults with ID over time. Therefore, initiating incremental changes to food habits with intermediate goals, e.g., changing one meal a day or initially replacing one or two unhealthy food items, may be suggested. There have been many health and weight interventions for adults with ID; however, extensive interventions tend to be ineffective (Harris et al., 2018), although often requested (Casey and Rasmussen, 2015).

The final attribute of the theory is observability, which addresses the degree to which the results of the innovation are visible (Rogers, 2003). Seeing positive results from changes in food habits encourages the permanent adoption of those changes (Fieldhouse, 2002). Fieldhouse noted that when changes in food habits are made for preventive purposes, the effects are not visible, and therefore, it is difficult to demonstrate benefit. However, the present respondents noted that adults with ID often have severe problems, and the physical effects of treatment need to have an observable impact on their health. An assessment instrument may facilitate longitudinal follow-ups. Gradual changes to food habits make it easier to achieve intermediate goals, which will inspire adults with ID to implement further changes.

According to Rogers (2003), the diffusion of innovation theory allows the adopter of the innovation to be active in the process. This means that success in the innovation of changing food habits for an adult with ID requires communication between the RD, the individual and the supporting staff to realize a mutual understanding regarding the situation. Rogers stated that when adopters actively participate in tailoring an innovation to fit their special situation, and when the innovation is adjusted, it will be easier to adopt and sustain the innovation (ibid). This flexibility also allows adults with ID and their supporting staff to take part in shaping the RD's advice for easier and more sustainable adoption. Participating in the solutions that influence one's life is imperative for all people, including adults with ID (Umb Carlsson and Adolfsson, 2018).

There is no register of RDs in Sweden, thus there is a risk that not everyone who met the inclusion criteria was identified. Finding relevant RDs was complicated and time consuming, which may be one reason why contact with RDs by adults with ID was often not made till 
problems were severe. Nevertheless, the 53 respondents represented primary care, habilitation centres and municipalities from different parts of Sweden. They provided exhaustive answers to the open-ended questions and comments to the multiple-choice questions. Thus, the amount of information provided was considered sufficient for the aim of the study.

As the present study has a qualitative approach, the credibility, transferability, conformability and dependability of findings should be considered (Bryman, 2008). A studyspecific questionnaire was chosen to ensure credibility. Further, a brief report of the results was discussed with a Nordic network of habilitation RDs. Transferability was addressed by presenting the respondents' professional specialties. However, the present study was conducted in Sweden, which may limit transferability to other contexts. Dependability was established by discussing the questionnaire with other researchers. Further, all the authors participated throughout the analysis process. According to Malterud (2012), the condensation process protects the systematic review of the sorted meaning units and prevents researcher bias. Conformability was reached by describing the research process and by reporting authentic quotations from the questionnaire responses.

In conclusion, the following practical implications emerge from this study:

- It is necessary to provide adults with ID and their supporting staff with individually tailored nutritional information.

- Adults with ID should be actively involved in lifestyle changes that affect their everyday lives.

- The RD must be included in the interdisciplinary team surrounding the adult with ID.

- If a new practice, including health-promoting service and support, is to be implemented, it needs to be compatible with the existing values of the staff and the adult with ID. In addition, it needs to be feasible for implementation in the everyday life of the individual.

Additionally, the present study identified several barriers that should be overcome in relation to the preparation of Swedish RDs to advise adults with ID about nutritional health issues. A systematic structure, knowledge about nutrition and knowledge about 
adults with ID and their living situations are needed. An assessment instrument may meet health promotion needs and facilitate longitudinal follow-ups of nutritional problems.

\section{References}

Adolfsson, P., Fjellström, C., Lewin, B. and Mattsson Sydner, Y. (2012), “Foodwork among people with intellectual disabilities and dietary implications depending on staff involvement", Scandinavian Journal of Disability Research, Vol. 12 No. 1, pp. 40-55.

Adolfsson, P., Mattsson Sydner, Y., Fjellström, C., Lewin, B. and Andersson. A. (2008), "Observed dietary intake in adults with intellectual disability living in the community", Food \& Nutrition Research, Vol. 52 No. 1. DOI: 10.3402/fnr.v52i0.1857

Bryman, A. (2008), Social Research Methods, Oxford University Press, Oxford.

Casey, A.F. and Rasmussen R. (2015), "Obesity", in Matson J.L. and Matson M.L. (Eds), Comorbid Conditions in Individuals with Intellectual Disabilities, Springer, New Your, NY, pp. 275-98.

Cheetham, T. and McMillan S. (2017), "Physical health", in Wehmeyer, M.L., Brown, I., Percy, M., Shogren, K.A. and Fung W.L.A. (Eds), A Comprehensive Guide to Intellectual \& Developmental Disabilities 2nd ed., Paul. H. Brookes Publishing, Baltimore, MD, pp. 665-78.

Davies, R. and Lennox, N. (2014), "The role of healthcare professionals", in Taggart L. and Cousin, W. (Eds) Health Promotion for People with Intellectual and Developmental Disabilities, Berkshire Mc Graw Hill Education, Open University Press, New York, NY, pp. 194203.

Devine, C.M., Jastran, M. and Bisogni C.A. (2004), "On the front line: practice satisfactions and challenges experienced by dietetics and nutrition professionals working in community settings in New York state", Journal of the American Dietetic Association, Vol. 104 No. 5, pp. 787-92.

Fieldhouse, P. (2002), Food and Nutrition, ( $2^{\text {nd }}$ ed.), Nelson Thornes, Cheltenham.

Harris, L., Melville, C., Murray, H. and Hankey, C. (2018), "The effects of multi-component weight management interventions on weight loss in adults with intellectual disabilities and obesity: a systematic review and meta-analysis of randomised controlled trials", Research in Developmental Disabilities Vol. 72, pp. 42-55.

Humphries, K., Rosenzweig, L., Cushing, P. and Licitra, R. (2012), "Registered Dietitians' Service to Group Homes for Adults with Developmental Disabilities." The open Nutrition Journal, Vol 6 pp. 116-22 available at: https://scholarworks.umt.edu/ruralinst independent living community participation/42 
Humphries, K., Traci M. A. and Seekins, T. (2009), "Nutrition and adults with intellectual or developmental disabilities: systematic literature review results", Intellectual and Developmental Disabilities, Vol. 47 No. 4, pp. 163-185.

Malterud, K. (2012), "Systematic text condensation: a strategy for qualitative analysis", Scandinavian Journal of Public Health, Vol. 40 No. 8, pp. 795-805.

Marks, B. and Sisirak, J. (2014), "Health promotion and people with intellectual disabilities", in Taggart L. and Cousins W. (Eds.), Health Promotion for People with Intellectual and Developmental Disabilities, Open University Press/McGraw-Hill Publisher, Maidenhead, pp. 17-29.

Ng, N., Wallén, E. F. and Ahlström, G. (2017), "Mortality patterns and risk among older men and women with intellectual disability: a Swedish national retrospective cohort study", BMC Geriatrics, Vol. 17 No. 1, pp. 1-13.

Ptomey, L. T. and Wittenbrook W. (2015), "Position of the Academy of Nutrition and Dietetics: Nutrition Services for Individuals with Intellectual and Developmental Disabilities and Special Health Care Needs", Journal of the Academy of Nutrition and Dietetics, Vol. 115 No. 4, pp. 593-608.

Reja, U., Manfreda, K.L., Hlebec, V. and Vehovar, V. (2003), “Open-ended vs. close-ended questions in web questionnaires", in Freligoj, A. and Mrvar, A. (Eds), Developments in Applied Statistics, (Metodološki zvezki), University of Ljubljana, Ljubljana, pp. 159-77.

Rogers, E.M. (2003), Diffusion of Innovations (5th ed.), Free Press, New York, NY.

Umb Carlsson, Ö. and Adolfsson P. (2018), "Quality of life is to experience adult social status", Journal of Policy and Practice in Intellectual Disabilities, Vol. 15 No. 4, pp. 272-83.

Umb Carlsson, Ö. and Sonnander, K. (2005), “A comparison of living conditions of adults with intellectual disabilities in a Swedish County and in general population", Journal of Policy and Practice in Intellectual Disabilities, Vol. 2 No. 3-4, pp. 240-8. 